Document downloaded from:

http://hdl.handle.net/10251/166339

This paper must be cited as:

Naskar, D.; Singh, SR.; Kumar, D.; Nandi, S.; Onaindia De La Rivaherrera, E. (2020). Emotion Dynamics of Public Opinions on Twitter. ACM Transactions on Information Systems. 38(2):1-24. https://doi.org/10.1145/3379340

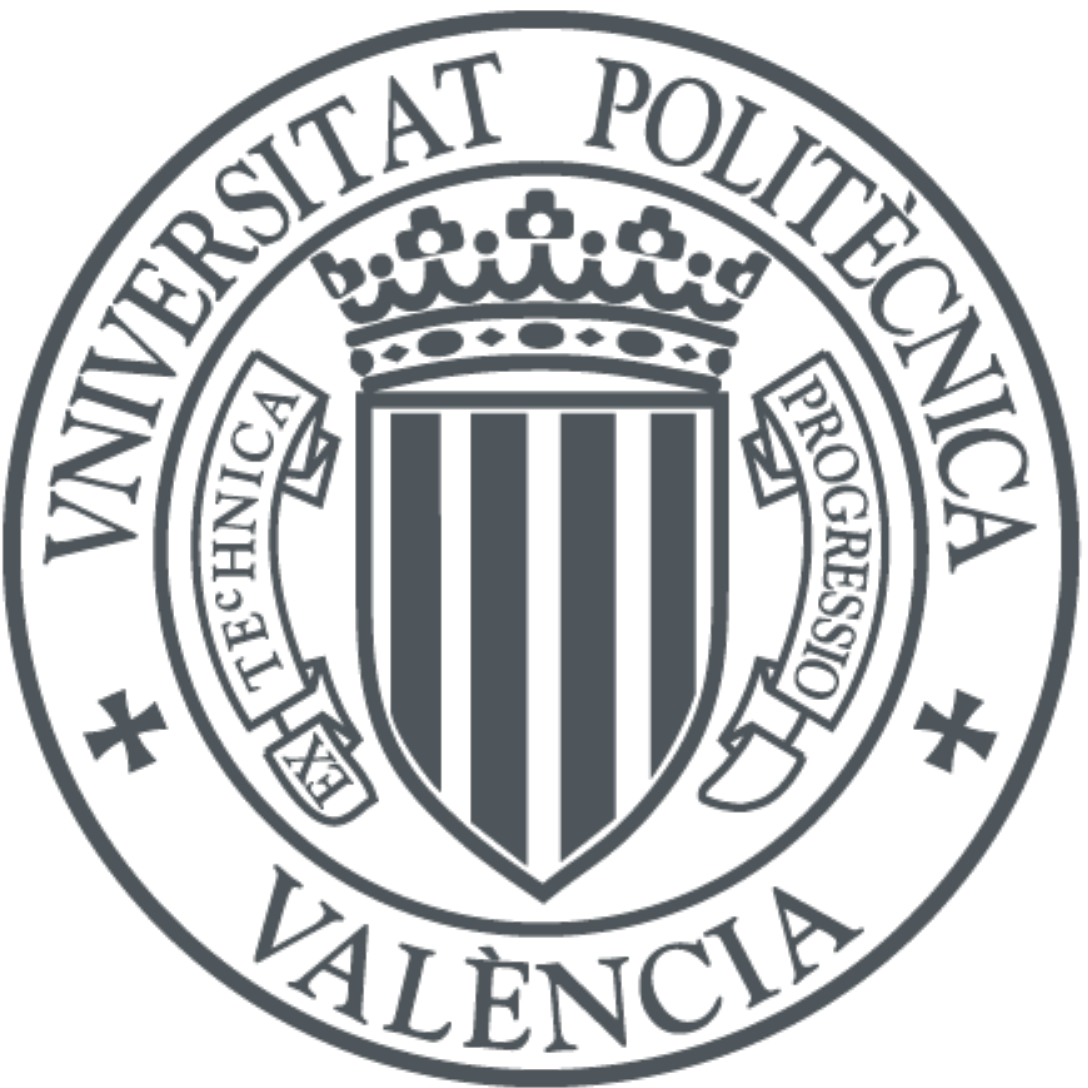

The final publication is available at

https://doi.org/10.1145/3379340

Copyright Association for Computing Machinery

Additional Information 


\title{
Emotion Dynamics of Public Opinions on Twitter
}

\author{
DEBASHIS NASKAR, Universitat Politécnica de Valéncia, Spain \\ SANASAM RANBIR SINGH, DURGESH KUMAR, and SUKUMAR NANDI, Indian Institute of \\ Technology Guwahati, Assam \\ EVA ONAINDIA DE LA RIVAHERRERA, Universitat Politécnica de Valéncia, Spain
}

\begin{abstract}
Recently, social media has been considered as the fastest medium of information broadcasting and sharing. Considering the wide ranges of applications such as viral marketing, political campaigns, social advertisement, etc., a study on influencing characteristics of users or tweets have attracted several researchers. It is observed from various studies that influential messages or users create a high impact on a social ecosystem. In this study, we assume that public opinion on a social issue on Twitter carries a certain degree of emotion, and there is an emotion flow underneath the Twitter network. In this paper, we investigate social dynamics of emotion present in user's opinion and attempt to understand (i) changing characteristics of user's emotion toward a social issue over time, (ii) influence of public emotions on individual's emotion, (iii) causing of changing opinion by social factors etc. We study user's emotion dynamics over a collection of 17.65 million tweets with $69.36 \mathrm{~K}$ users and observe $63 \%$ of the users likely to change their emotional state against the topic into their subsequent tweets. Tweets coming from the member community have higher influencing capability to others than the other sources. It is also observed that retweet influences users more than hashtag, mention and reply.
\end{abstract}

CCS Concepts: • Information systems $\rightarrow$ Sentiment analysis; • Networks $\rightarrow$ Social media networks; Network dynamics.

Additional Key Words and Phrases: Emotion transition, influence measure, opinion discussion, social agreement, social dynamics

\section{INTRODUCTION}

With the explosive growth in its popularity, social networking sites like Facebook, Twitter, etc. have become an important medium for people to acquire and share information. People often tend to rely on these platforms for retrieving information about topics of their interest, and often make their decisions/opinions based on the acquired information. Social network sites are used in various tasks such as political campaigns [Gu et al. 2013; Stieglitz and Dang-Xuan 2013], social advertisement [Li and Shiu 2012], social aspects of emotions [Kim et al. 2012], expert finding [Pal and Counts 2011], viral marketing etc [Bi et al. 2014; Chen et al. 2010; Ding et al. 2013; Shi et al. 2018; Sun and Ng 2013] for influencing people. Different studies give credits the success of Arab spring [Wolfsfeld et al. 2013], Brazilian protests [Costa et al. 2015], Nirbhaya justice [Ahmed et al. 2017] etc. to social networking platforms. Social movements are believed to be highly influenced by social media sites, particularly in their organization and communication. Do social media posts about current events, news, and sociopolitical debates influence people's opinions? This is one of the core questions that many of the studies on social media data analysis are attempting to understand. Several studies [Kwak et al. 2010; Weeks et al. 2017] observe that social activities and interactions greatly effect people's day-to-day activities, lifestyle, reading habit etc. In regards to political and social issues, public policies, studies show different observations. Based on the finding in the study [Karakiza 2015], what people say or post on social media highly influence one's support on public policies. The same is also found to be true for political leaders while supporting or opposing a public policy. Study [Cha et al. 2010] also noted that the influence pattern is different for different countries and leaderships. However, has social media activities on a topic or news story ever changed one's opinion on a political issue? Study [Ziegler and Lausen 2005]

Authors' addresses: Debashis Naskar, debashis@drtc.isibang.ac.in, Universitat Politécnica de Valéncia, Spain; Sanasam Ranbir Singh, ranbir@ iitg.ernet.in; Durgesh Kumar, k.durgesh@iitg.ac.in; Sukumar Nandi, sukumar@iitg.ernet.in, Indian Institute of Technology Guwahati, Assam; Eva Onaindia de la Rivaherrera, onaindia@dsic.upv.es, Universitat Politécnica de Valéncia, Spain. 
analyzed propagation of trust and distrust on social networks, what can be considered the first paper in which sentiment propagation was studied. Interesting conclusions, like that positive and negative sentiments follow a different propagation pattern [Hillmann and Trier 2012], have been drawn from the various investigations on sentiments in social networks. Other works studied the correlation between emotions and information diffusion, finding that those messages emotionally charged were re-tweeted more often [Stieglitz and Dang-Xuan 2013], or investigated if the topic and the opinion of the user's contacts affect the own user's opinion [Tang and Fong 2013]. Motivated by the above studies, this paper investigates changing characteristics of people's opinion against an event on Twitter, and how do mass discussions/interactions influence in changing one's opinion against an event. We use the emotion of a user reflected in the post as the matrix to indicate his/her opinion in support/oppose a social event.

This study focuses on emotion dynamics of a user while posting comments against an event/topic from three different social characteristics: i) emotional (excitement, contentment, depression and distress), ii) community (follower followee , membership relationship), and iii) conversational (tweet, retweet, mention, reply). Identifying influential features can help us to understand factors causing people to change their opinions and in turn help agencies like advertisers and marketers to design more effective campaigns. This paper systematically explores user's changing characteristics of emotion over time, and attempts to find answer to the following three questions.

- Do people change their opinion against an event/issue over time?

- Which type of opinions against which type of events are more pron to change?

- If people change their opinion, which of the social factors cause them to change their opinion?

To investigate the above questions, we collect posts related to twelve different events from Twitter using Search-API. It consists of $12.91 \mathrm{~K}$ users over a total of $17.65 \mathrm{M}$ tweets. Emotional states of the users reflected in the post are determined for each tweet using Russell's model of affect which correctly classifies the emotions expressed in over $90 \%$ of text messages [Hasan et al. 2014]. For each user against a topic/event, Temporal Emotional State Chain (TESC) is prepared. Details of the data preparation is given in Section 3. All the experimental analysis are conducted over the collection of TESCs across different users and different events. From various experimental setups, this paper makes the following contributory observations.

- We show that $63 \%$ of the user change their opinions and if an individual shares positive emotion against a topic, (s)he is likely to stay in the same emotion state in his/her subsequent tweets.

- Tweets coming from a member community have higher influential ability to an individual than the other sources like followers.

- Retweeted tweets can also influence a user higher than the tweets received through hashtag, reply and mention.

The rest of this paper is organized as follows. Section 2 review the related works. Section 3 describes how we collected the dataset and labeled by sentiments as well as a brief note on Russell's model. Section 4 shows the emotion transition on Twitter. Section 5 explains the process of understanding influence of incoming tweet. Section 6 explains about different characteristics during emotion state transition. The last section concludes our work.

\section{RELATED WORK}

This section briefly reviews the earlier literature that exploits different characteristics of opinion dynamics and measuring user influence in social media. In Twitter, the number of studies have been conducted on changing opinion and discussion on user influence. A number of researchers have examined how message content affects individual retweeting decisions. They show users can influence brand content diffusion via retweets [Araujo et al. 2017], the role of content influence on social media via retweets behavior [Zhang et al. 2017]. Authors in 
the study [O'Connor et al. 2010] measures of public opinion derived from polls with sentiment measured from analysis of text from the popular microblogging site Twitter.

\subsection{Social Behavioral Aspect}

Emotional contagion has an influence on individual and group-level communication behavior in terms of information coordination and sharing [Suh et al. 2010a]. In the same direction, [Ferrara and Yang 2015] conducted a study on the dynamics of emotional contagion using a random sample of Twitter users and measure the emotional valence of content the users are exposed to before posting their own tweets. A high level of cognitive involvement such as anger, anxiety, awe, or amusement might also trigger a high level of physiological arousal, whereby low arousal or deactivation is characterized by relaxation and high arousal or activation is characterized by activity [Berger 2011]. Social networking is a multidimensional concept where users share a different type of behavioral aspect [Stieglitz and Dang-Xuan 2013] over a topic. An individual emotion on behavioral concepts possible to utilize through social networks in viral marketing. In this part, we study different emotions transitions of a user shows in social networks and also a different type of emotion detection [Colneriĉ and Demsar 2018]. [Myers et al. 2012] represents a model in which information can reach a node via the links of the social network or through the influence of external sources. The model used to infer the quantify the external influences over time and describe how these influences affect information adoption. Compare with others study, we consider four regions which are based on 16 emotions of Russell's model of affect. This emotion model combined with two main dimensions (i.e. valence and arousal) in a $2 \mathrm{D}$ circular space.

The emotion transition leads to find out how likely a user express their emotion after receiving a tweet responding. A study conducted by authors in [Kim et al. 2012] where they show social aspects of the user's emotion by using Plutchik's wheel model and also examine that the conversational partners can influence each others' emotions and topics. [Bollen et al. 2011] investigate collective public mood states derived from large-scale collections of daily Twitter posts over time. They analyze tweets by using two mood tracking tools, namely OpinionFinder and also 6 dimensions mood measures Google-Profile of Mood States (GPOMS). Similarly, [Amalanathan and Anouncia 2017] attempts to study the nine basic human emotions and their significance in various social network activities to determine the right strategies of marketing in e-business.

\subsection{Social Influential Characteristics}

Understanding influential factors is an important task to understand dynamics in user's opinion on social network. A comprehensive comparison of different influential factors (indegree, retweets and mentions) on users' social dynamics is studied in [Cha et al. 2010]. A similar study is conducted by [Ye and $\mathrm{Wu}$ 2010] where they measure propagation patterns of tweet messages and social influence by following three metrics, i.e. follower, reply and retweet. [Peng et al. 2017] examined a set of different characteristics (Dynamic, Propagative, Composable, Measurable, Subjective, Asymmetric and Event-sensitive) to understand user's dynamics and identify influential users on Twitter. [Kwak et al. 2010] study information diffusion pattern of topological features namely singleton, reply, mention and retweet. They also study the temporal behaviour of trending topics. Unlike above studies, [Kim et al. 2012] explores the affect of social and conversational characteristics of users on emotional dynamics. Specifically, they look into the social conversational features that lead to transition of emotion states within a discussion chain. [Shi et al. 2018] propose a theoretical framework to systematically investigate the determinants of individual dissemination behavior in a Twitter network. They found information related to topical preference and homophily value are most influential on individual dissemination behavior.

From the above discussion, we observe that while a large number of studies have been conducted to study the community channel, not many of them study the relationship between the community channel with the emotional aspect of the tweets. Few studies [Cha et al. 2010; Kim et al. 2012] that have been conducted in this 
direction do not consider a very wide ranges of characteristics. This study in particular considers the effect of a wide ranges of community and conversational characteristics on the emotion dynamics of a user while posting their opinion. Our experiment is completely based on Twitter datasets and all the possible sources are accommodated into this analysis. A popular study [Cha et al. 2010] defined that the majority of the people are influenced by three important activities such as followers influence, retweets influence and mentions influence. Including these three sources of influence, our study also covers some more extra parameters of influences such as hashtag tweets, replies, member lists etc. Our finding shows that the member-list is one of the important community channels which shows more influential to the user and retweets is more influential characteristics among others.

\subsection{Social Influence Evaluation Measure}

Identifying influential users is an important aspect in social media related studies. Identifying influential users can aid in tasks like social or political campaigns or viral marketing etc. In this direction, [Zhang et al. 2017] study the influence of content as well as users on the rebroadcasting pattern of a message. They observe that along with the content of a message, the rebroadcasting of a message by a user is also dependent on other users and the relevance of the message to the user. A similar study was conducted by authors in [Araujo et al. 2017] where they study the influence of users in the diffusion of information in a Twitter network. [Weng et al. 2010] proposed TwitterRank algorithm, an extension of PageRank algorithm to find the influential users in a Twitter network for a given topic. [Kwak et al. 2010] propose different measures for ranking influential users and report a comparison among them. [Ding et al. 2015] proposed a novel random walk model to measure the users' influence. For measuring a user's influence, they take into account not only the follower network of the user but also the popularity of the tweets. A method for measuring user influence is also proposed in [Zhang et al. 2016]. This paper presents TrueTop, the first sybil-resilient system to measure the influence of Twitter users. ProfileRank, a random walk based method inspired by PageRank is proposed in [Silva et al. 2013] to find influential users and relevant content.

Rather than finding influential users, [Saez-Trumper et al. 2012] propose a method to find trendsetters in information networks. Trendsetters are different from other influential users in that they need not necessarily be famous but are able to spread a new idea over a social network successfully. While all of the above studies have considered Twitter as the experimental framework, [Liakos et al. 2016] investigates the influence mechanisms in Pinterest social media platform. Another influence study conducted by [Nguyen et al. 2017], where author propose the computation of Influence Spectrum algorithm for seeking a set of influential people on several social networks such as NetHEPT, NetPHY, Epinions, DBLP and Twitter. Similarly, [Wang et al. 2018] define two influence maximization queries to track influential users over Twitter and Reddit datasets. Other than the community structure of a network, [Vardasbi et al. 2017] propose a linear-time shell decomposition method based on the layer structure to maximize the influence in large scale networks. Their method can explain the different behaviors of real networks and predict the saturation dynamics in the networks.

\section{DATA PREPARATION AND EMOTIONAL MODELING}

This section describes the experimental dataset used in this study. For our purpose of analyzing real-time events, we chronologically retrieved tweets through Twitter Search-API ${ }^{1}$ and created our own datasets. We collected tweets related to different events that contain a specific hashtag. Our objective is to analyze the sentiments derived within a conversation that occurs in Twitter and investigate how the emotions are changed dynamically across the users that take part in the conversation. Datasets are categorized based on different events happening in the world related to policy, movie, accident, terrorism and sport. We tried to address different types of topic

\footnotetext{
${ }^{1} \mathrm{http}: / /$ twitter4j.org
} 
Table 1. Size of the all datasets

\begin{tabular}{|c|c|c|c|c|c|c|c|}
\hline Event/Topic & Categories & Starting Date & Ending Date & $\begin{array}{l}\text { Total \# of } \\
\text { Tweets }\end{array}$ & $\begin{array}{c}\text { Total \# of } \\
\text { Sent Tweets }\end{array}$ & \# of Users & $\begin{array}{c}\text { Avg \# of } \\
\text { Sent Tweets }\end{array}$ \\
\hline \#BlackMoneyDebate & Policy & $\begin{array}{c}14-11-2016 \\
09: 10: 56\end{array}$ & $\begin{array}{c}27-11-2016 \\
16: 51: 03\end{array}$ & 616343 & 15936 & 1260 & 12.6 \\
\hline \#Brexit & Policy & $\begin{array}{c}18-07-2016 \\
13: 29: 26\end{array}$ & $\begin{array}{c}24-07-2016 \\
14: 58: 39\end{array}$ & 686434 & 17053 & 2688 & 6.3 \\
\hline \#AlienCovenant & Movie & $\begin{array}{c}16-05-2017 \\
14: 20: 16\end{array}$ & $\begin{array}{c}20-05-2017 \\
12: 21: 33\end{array}$ & 74286 & 4957 & 1504 & 3.3 \\
\hline \#Baahubali2 & Movie & $\begin{array}{c}08-05-2017 \\
21: 44: 17\end{array}$ & $\begin{array}{c}10-05-2017 \\
11: 05: 39\end{array}$ & 53391 & 2750 & 679 & 4.0 \\
\hline \#BadmintonRio2016 & Sport & $\begin{array}{c}13-08-2016 \\
19: 11: 38\end{array}$ & $\begin{array}{c}23-08-2016 \\
12: 24: 54\end{array}$ & 66679 & 7413 & 784 & 9.4 \\
\hline \#UCLFinal & Sport & $\begin{array}{c}04-06-2017 \\
07: 32: 05\end{array}$ & $\begin{array}{c}11-06-2017 \\
15: 21: 09\end{array}$ & 100547 & 7481 & 1996 & 3.7 \\
\hline \#SyriaGasAttack & Terror Attack & $\begin{array}{c}06-04-2017 \\
04: 35: 33\end{array}$ & $\begin{array}{c}07-04-2017 \\
04: 19: 38\end{array}$ & 10823 & 1477 & 557 & 2.6 \\
\hline \#StockholmAttack & Terror Attack & $\begin{array}{c}07-04-2017 \\
15: 24: 49\end{array}$ & $\begin{array}{c}12-04-2017 \\
18: 13: 00\end{array}$ & 2092 & 375 & 128 & 2.9 \\
\hline \#GrenfellTower & Accident & $\begin{array}{c}15-06-2017 \\
10: 59: 25\end{array}$ & $\begin{array}{c}17-06-2017 \\
23: 34: 56\end{array}$ & 136821 & 6499 & 2297 & 12.8 \\
\hline \#UnitedAirlinesAssault & Accident & $\begin{array}{c}10-04-2017 \\
16: 17: 14\end{array}$ & $\begin{array}{c}13-04-2017 \\
15: 43: 57\end{array}$ & 7176 & 7481 & 583 & 2.7 \\
\hline \#MacronPresident & Politics & $\begin{array}{c}08-05-2017 \\
06: 38: 01\end{array}$ & $\begin{array}{c}10-05-2017 \\
22: 57: 53\end{array}$ & 6171 & 643 & 235 & 2.7 \\
\hline \#Trumpregrets & Politics & $\begin{array}{c}13-03-2017 \\
17: 16: 29\end{array}$ & $\begin{array}{c}23-03-2017 \\
12: 11: 11\end{array}$ & 4884 & 768 & 201 & 3.8 \\
\hline
\end{tabular}

to check whether the emotion of the user act differently or not. All the tweets were collected in the english language. This study considers twelve events/topics belonging to six different categories as shown in Table 1 . Some of the hashtags representing the events/topics are manually identified. These Hashtags are further used to collect the related post. The collected tweets mainly contain the following information (i) user information i.e., the user who posted the tweet (ii) tweet text (iii) type of tweet i.e., direct tweet, retweet, reply, quoted tweet (iii) time of posting the tweet. To study opinion dynamics, one should post at least two tweets. We therefore first identify users who have posted at least two tweets against a topic. The dataset consists of about 17.65 million tweets, and $69.36 \mathrm{~K}$ number of users. Out of the total number of tweets, $72.83 \mathrm{~K}$ number of tweets sent by $12.91 \mathrm{~K}$ users and the rest of the tweets (i.e. 10.36 million) have been received by the same $12.91 \mathrm{~K}$ users. Since our study focuses on the opinion dynamics of these users only, therefore we require users to have sent at least two tweets within a two-time frame. It means, out of total users, $12.91 \mathrm{~K}$ users have posted at least two number of tweets. The description of the datasets are given below:

- \#Blackmoneydebate comprises the tweets of the user that are related to demonetization which started in India from November 10 till December 30, 2016.

- \#Brexit tweets are about the referendum about the United Kingdom's withdrawal from the European Union held on June 23, 2016.

- \#AlienCovenant is an American science fiction horror film directed by Ridley Scott. It was released in the United States on May 19, 2017.

- \#Baahubali2 is an Indian historical fiction film that was theatrically released over 9000 screens worldwide on 28 April 2017.

- \#BadmintonRio2016 comprises the tweets of the user about the final championship of badminton in Rio2016.

- \#UCLfinal is about the 2017 UEFA Champions League Final football tournament between the Italian side Juventus and Spanish side Real Madrid which played at Millennium Stadium in Cardiff, Wales on 3 June 2017. 


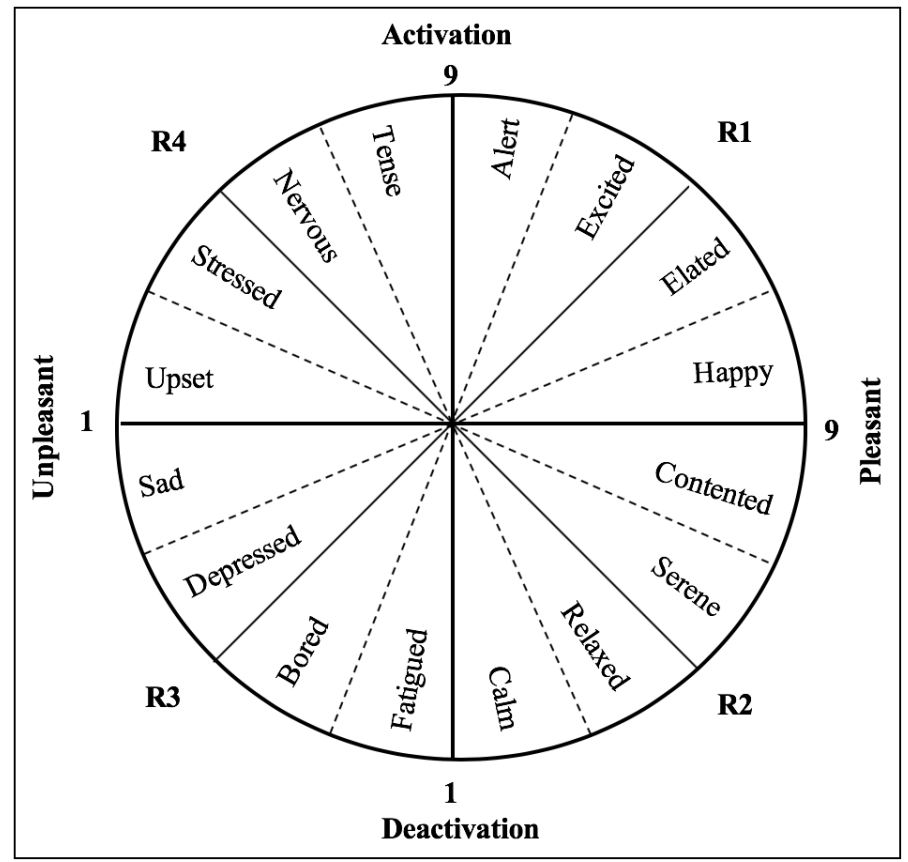

Fig. 1. Russell's Model of Affect [Feldman Barrett and Russell 1998]

- \#SyriaGasAttack is about a gas attack in north-western Syria where more than 80 people were killed on April 4, 2017. Survivors and aid workers shared their stories of horror and shock after a suspected chemical attack in Syria.

- \#StockholmAttacks tweets are related to attack which happened in Stockholm, the capital of Sweden on April 7, 2017. A hijacked truck was deliberately driven into crowds and killed four people, including many more injured.

- \#GrenfellTower is a 220-foot high tower block of public housing flats in North Kensington, west London. Collected tweets are about the Grenfell Tower fire on 14 June 2017 which caused at least 80 deaths and over 70 injuries.

- \#UnitedAirlinesAssault is about an Asian passenger Dr. David Daoa who was violently dragged off by security officers from an overbooked United Airlines flight on April 10, 2017.

- \#MacronPresident is a French politician who won the second round of the presidential election on 7 May 2017.

- \#Trumpregrets is a conversation about those American citizens who voted for Trump and now regret their decision.

\subsection{Data Pre-processing}

For each participating user, we extract and arrange the tweets posted by the user in the order of posting time. As mentioned above, we assign an emotional state to each of the selected tweets to enable us to investigate a user's emotion dynamics while participating in social discussions. The details of the data preparation are discussed below. 


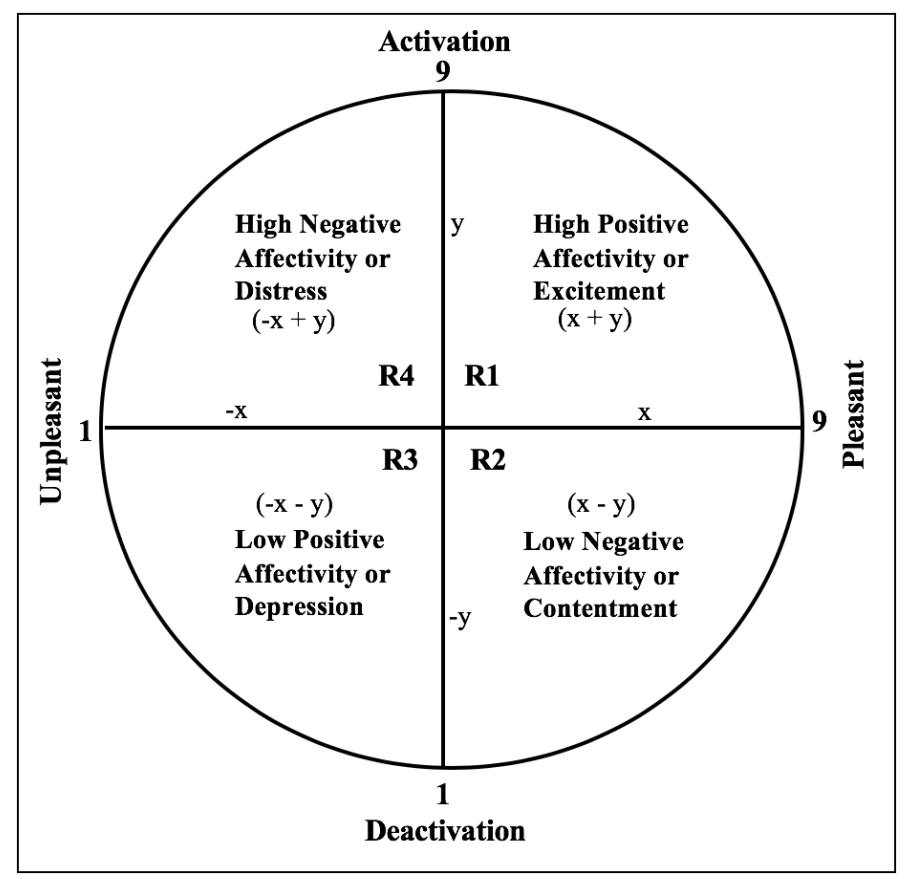

Fig. 2. A Semantic Structure of Affect. The letters $x$ and $y$ represent semantic components: $x=$ Pleasant; $y=$ Activation. [Feldman Barrett and Russell 1998; Yik et al. 1999]

3.1.1 Russell's Model of Affect. We use the well known sixteen state Russell's circumflex model of affect [Feldman Barrett and Russell 1998] to estimate the state of emotion present in a given tweet ${ }^{2}$. According to this model, every affective experience is defined by valence and arousal coordinate in the 2D circumflex shown in Figure 1 . A numerical value for valence ranges from 1 (unpleasant) to 9 (pleasant) and arousal ranges from 1 (deactivation) to 9 (activation). The emotional state or sentiment label of a given entity (message or user) has been formed according to its valence ( $x$-axis) and arousal ( $y$-axis) values. Figure 2 shown on the right-hand side are the more pleasant states (+ve $x$ ); on the left-hand side the more unpleasant ones (-ve $x$ ). The upper half shows the more activated states (+ve $y$ ), the lower half the more deactivated ones (-ve $y$ ). To reduce the number of emotional states, we consider four quadrants defined in [Russell 1980] and named them as excitement (R1), contentment (R2), depression (R3) and distress (R4). Excitement is a state with high positive affect $(x+y)$, while contentment is a state with low negative affect $(x-y)$. Similarly, distress is a state with high negative affect $(-x+y)$ and depression is a state with low positive affect $(-x-y)$ [Feldman Barrett and Russell 1998; Yik et al. 1999].

3.1.2 Finding Emotional State of a Tweet. To determine the emotional state of a tweet using Russell's circumflex model, we first need to estimate the valence and arousal score of the tweet. The aim of sentiment extraction is to compile sentiment words. One of the most efficient approaches for this purpose is the dictionary-based approach. Dictionary-based approaches use dictionaries of emotional words which are associated with a sentiment score. To estimate the valence and arousal score of a tweet, we use the ANEW dictionary of affect [Bradley and Lang 2010]. The new version of the Affective Norms for English Words (ANEW) dictionary [Nielsen 2011] is being developed to provide the mean and standard deviation of normative emotional ratings (valence $v$ and arousal

${ }^{2}$ This is the extension of original eight state Russell's model [Russell 1980] 
a) for 2477 unique words in English. The word list of ANEW initiated from a set of obscene words as well as a few positive words. Later on, different slang words were included such as WTF, LOL and ROFL. The entries of this dictionary match by applying Porter word stemming and WordNet lemmatization. It also contains another less strongly-related dimension was called dominance. However, for the purpose of our experiment, we only concentrate on two primary dimensions.

The performance of the dictionary-based approach can be evaluated according to two aspects: 1) the number of emotional words covered by the dictionary and 2) the nature of sentiment score provided by the dictionary. ANEW computes sentiment score with the valence and arousal values of the word, which range from 1 to 9 . ANEW allows us to calculate a more accurate sentiment value which fits better our aim of having a bi-dimensional representation of sentiments as well as to measure the intensity of expressed sentiments.

3.1.3 Sentiment Scores Calculation. We show an example of a tweet message that comprises three emotional words that exist in the ANEW dictionary along with their valences and arousal values.

Hazirah Afifah @AzieFifa (Fri Aug 19 19:11:27 CEST 2016): "Good job guys!!!! We Malaysian re so proud!!!!! MalaysiaBoleh badmintonRio2016".

- Good, $v=[\mu: 7.47, \sigma: 1.45], a=[\mu: 5.43, \sigma: 2.85]$

- job, $v=[\mu: 5.83, \sigma: 2.15], a=[\mu: 5.20, \sigma: 2.23]$

- proud, $v=[\mu: 8.03, \sigma: 1.56], a=[\mu: 5.56, \sigma: 3.01]$

The aim of this phase is to associate each entity $e$ with a tuple $\left(v_{e}, a_{e}\right)$. The average sentiment score of a tweet message $d$ is calculated with the valence and arousal of the stem words of $d$ that appear in the ANEW dictionary (emotional words of $d$ ). Then, the sentiment score of a user $u$ is calculated with the score of his/her tweet messages and we associate the corresponding sentiment label $S_{u}$.

The sentiment score of a user is calculated as the average emotional value of all the tweets sent by the user. For example, if we amalgamate the three words Good, job and proud of the above message $d$, the result of the weighted average formula (1) for the valence and arousal is $X_{d}=7.30$ and $Y_{d}=5.41$, respectively. We then use the mean points to determine the emotional state in the Russell's circumflex model, i.e., the coordinate $(7.30,5.41)$ falls in the excitement region $(R 1)$. In a given tweet, more than one emotional word may be present. Like in [Naskar et al. 2016], we use the formula defined in the equation 1 to determine the overall emotional state of a tweet.

$$
X=\frac{\sum_{i=1}^{N} \frac{\mu_{i}}{\sigma_{i}}}{\sum_{i=1}^{N} \frac{1}{\sigma_{i}}} \quad(X, \mu, \sigma)
$$

where $X$ is the mean value of valence (similarly, mean value of arousal), $N$ is the total number of emotional words within the message, $\mu$ is the word's mean value of valence (equivalently for arousal) and $\sigma$ is the word's standard deviation of valence (equivalently for arousal).

\subsection{Temporal Emotional State Chain}

This section describes formation of a Temporal Emotional State Chain (TESC). Our presumption is that a person's emotion depends on his/her personal opinion and the past history s/he received from neighbors by following different emotions, popularity and characteristics of messages. The Temporal Emotional State Chain is the sequence of outgoing and incoming tweets within two time frame. Every participating user's data is defined by the sequence of outgoing and incoming tweets arranged in the order of posting time. Outgoing tweets are those tweets posted by the target user about the topic under consideration. Whereas the incoming tweets are those tweets posted by other users about target topic, and are received by the user through one of the following : 


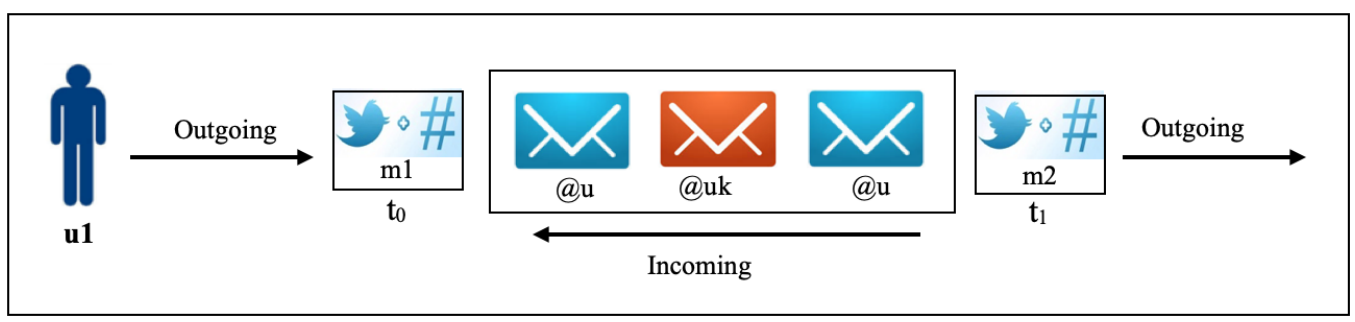

Fig. 3. Temporal Emotional State Chain

hashtag, mention, reply, retweet, following-list, member-list, other-list. We use the following terminologies to describe the chain formation.

- User $(u)$ : User $u$ is the current user for which we want to analyse the temporal emotional state chain.

- Incoming mention (InM) : If a tweet is posted by another user with @u, then this tweet is an incoming mention (InM) tweet to user $u$.

- Incoming retweet (InRT) : A quoted retweet with @u is referred to as incoming retweet (InRT) for user $u$.

- Incoming reply (InR) : An incoming reply (InR) to a user $u$ is direct reply to $u$ 's post.

- Incoming hashtag (InH) : A tweet bearing the same hashtag as that of $u$ 's tweet is the incoming hashtag (InH) for $u$.

- Incoming member-list (InML) : Any user $u$ of a group post a tweet and correspondingly another user post another tweet from the same group, we refer to this tweet as coming from incoming member-list (InML).

- Incoming following-list (InFL) : The user $u$ who is following someone and list of tweets bearing the same hashtag seen by followee ( $u$ who is being followed), we refer to these tweets as coming from incoming following-list (InFL).

- Incoming other-list (InOL) : Any user $u$ who is mentioned by someone but not associated with followee or member list, we refer to as a incoming other-list (InOL).

In TESC, we wanted to register the message that causes the reaction of a user and, at the same time, whether the user reacts to this message or not. Consequently, we needed to know the message received by the user and the possible message sent by the user. More specifically, we consider the time at which tweets were posted. Given a user $u$, we estimate the incoming tweets that the user $u$ has received between two consecutive tweets (i.e. $m_{0}$ and $m_{1}$ ) posted by $u$. The time of the two consecutive tweets sent by a user $u$ is referred to as $t_{0}$ and $t_{1}$, and the received messages of the tweets that were posted at a time between $t_{0}$ and $t_{1}$.

Given a user $u$ and a topic \#h, a typical temporal tweet chain is defined by the following tuple chain, where $\downarrow$ denotes incoming and $\uparrow$ denotes the outgoing tweets.

$<u, \# h>\rightarrow \quad<\ldots, \downarrow m_{t_{0-1}}^{c}>, \uparrow \mathbf{m}_{\mathbf{t}_{0}},<\downarrow m_{t_{0+1}}^{c}, . .>, \uparrow \mathbf{m}_{\mathbf{t}_{1}},<\downarrow m_{t_{1+1}}^{c}, \ldots>, \uparrow \mathbf{m}_{\mathrm{t}_{2}}, \ldots$

where $c \in\{$ in $H$, inRT, inR, inM, inFL, inML, inOL $\}$. When the user $u$ posts his first tweet at $t_{0}$ on topic \#h, public discussion on the topic $\# h$ might have already taken place. It is denoted by the tuple $\downarrow m_{t_{0-i}}^{c}, i=1,2, .$. and $c \in\left\{i n H\right.$, inRT, inR, inM, inFL, inML, inOL \}. Similarly, incoming tweets between the user's tweet $\uparrow m_{t_{k}}$ and $\uparrow m_{t_{k+1}}$, is denoted by the tuple $<\downarrow m_{t_{k+0}}^{c}, \downarrow m_{t_{k+1}}^{c}, \ldots>$. An example is also shown in Fig 3.

The emotional state of a tweet in a temporal tweet chain is determined using Russell's circumflex model of affect as described in section 3.1.2. If $R_{j}, j \in 1,2,3,4$ denotes one of the four emotional state for a given tweet, the above temporal tweet chain can be transformed into the following temporal emotional state chain.

$$
<u, \# h>\rightarrow \quad<\ldots, \downarrow R_{j, t_{0-1}}^{I n M}>, \uparrow \mathbf{R}_{\mathbf{j}, t_{0}},<\downarrow R_{j, t_{0+1}}^{I n H}, . .>, \uparrow \mathbf{R}_{\mathbf{j}, t_{1}},<\downarrow R_{j, t_{1+1}}^{I n H}, . .>, \uparrow \mathbf{R}_{\mathbf{j}, \mathbf{t}_{2}}, . .
$$

In all the experimental analysis reported in the subsequent section, we use the above temporal emotional state chain for each user. 


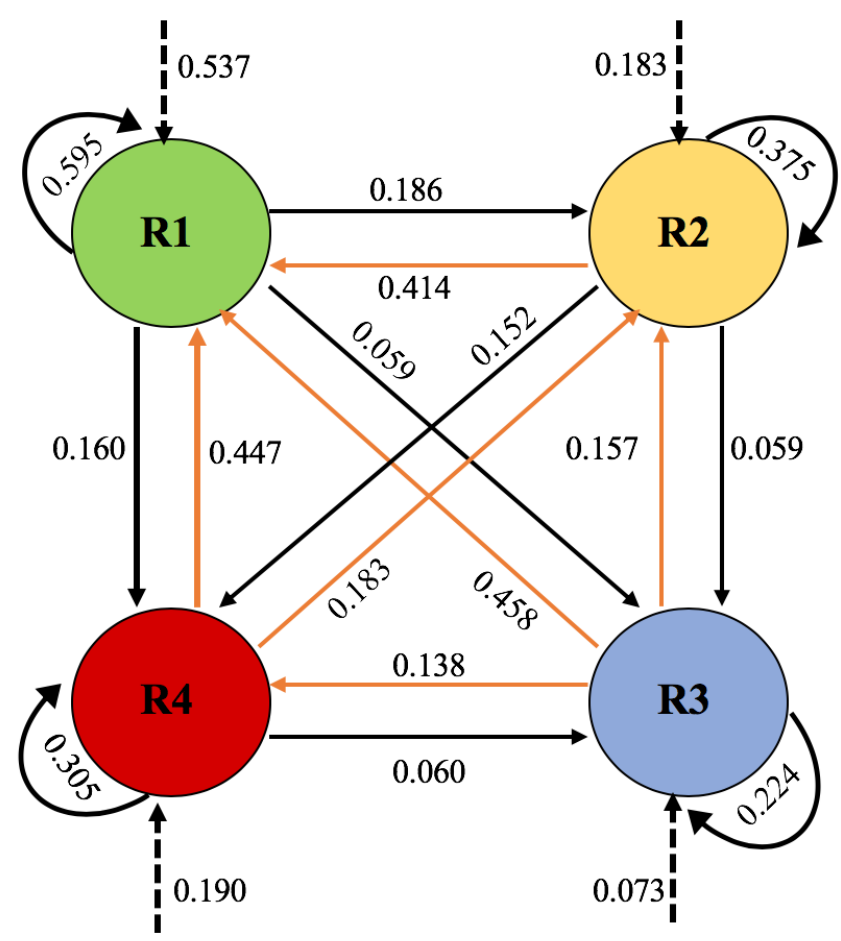

Fig. 4. Macro average transition probabilities over all topics. Initial state probability is represented by dot arrow.

\section{EMOTION TRANSITION ON TWITTER}

This section analyzes the characteristics of the emotional state transition of a user in his/her subsequent tweets against a topic. We focus on analyzing the following characteristics: (i) transition probability of users emotional state change in subsequent tweets, (ii) likely initial state of user's emotion while posting a tweet against a topic, (iii) relationship between user's emotional state and nature of the topic, and (iv) participation of the user into the network conversation and their frequency while transit from one state to another.

To perform the state transition of the user, a probabilistic sequence model, i.e. the Markov model is adopted. The simplest Markov model is the Markov chain [Andrieu et al. 2003]. According to the Markov model, the next state is solely chosen based on the current state. The transition probabilities control the way the hidden state at time $t$ is chosen given the hidden state at time $t-1$. The set of transition probabilities for transitions from any given state must sum to 1 . In this study, emotion states of users are likewise labeled with Russell's regions $S=R 1, R 2, R 3$ and $R 3$. The process starts in one of these states and moves successively from one state to another. Each move is called a step. If the chain is currently in state $s_{i}$, then it moves to state $s_{j}$ at the next step with a probability denoted by $p_{i j}$, and this probability does not depend upon which states the chain was in before the current state. The probabilities $p_{i j}$ are called transition probabilities. The process can remain in the state it is in, and this occurs with probability $p_{i i}$. Let $\langle R 1\rangle$ is a current emotion state of the tweets sent by the user at $t$, then next move can be towards $\langle R 2\rangle,\langle R 3\rangle$ or $\langle R 4\rangle$ or this transition can remain in the $\langle R 1\rangle$. However, the emotional state of the user can start with any of these four states. 


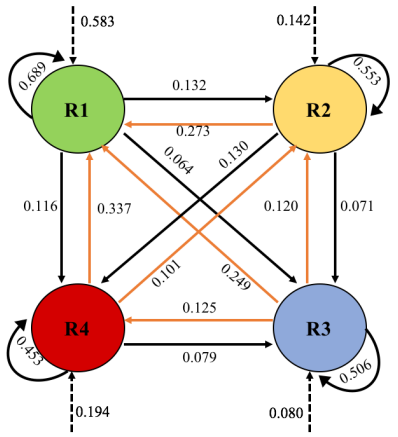

(a) Blackmoneydebate

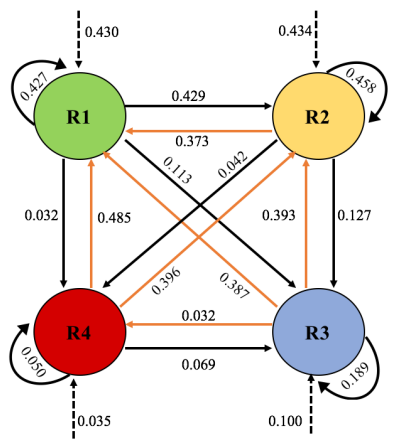

(d) Baahubali2

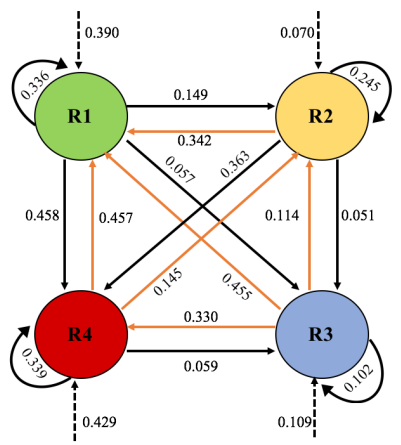

(g) SyriaGasAttack

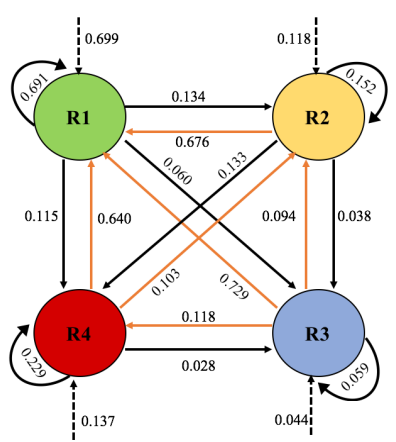

(j) UnitedAirlinesAssault

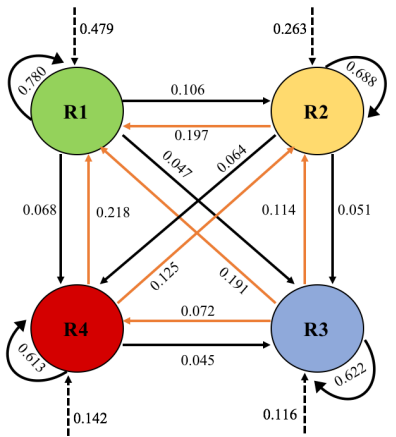

(b) Brexit

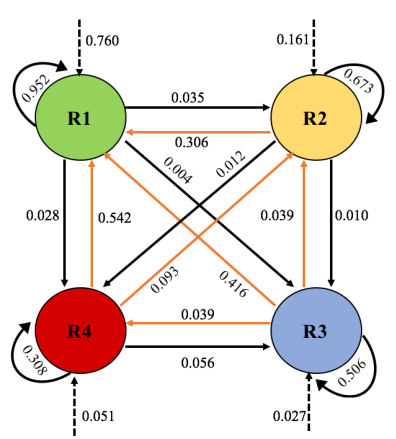

(e) BadmintonRio2016

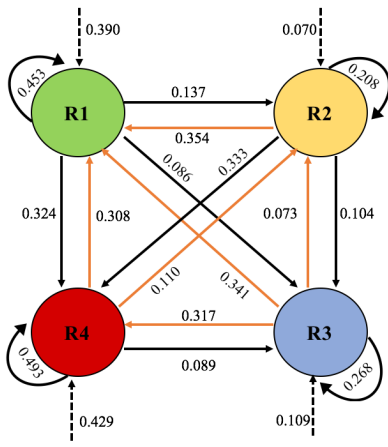

(h) StockholmAttacks

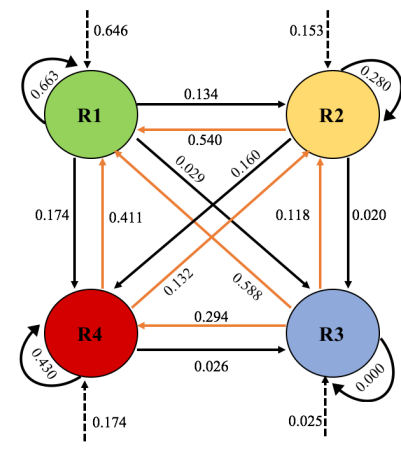

(k) MacronPresident

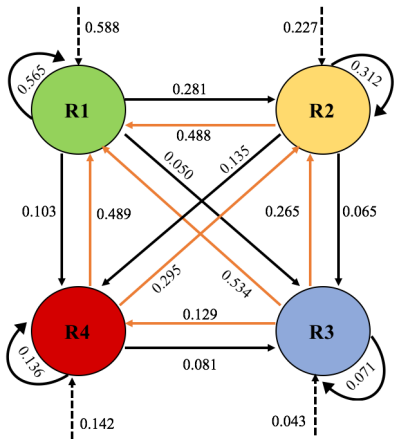

(c) AlienCovenant

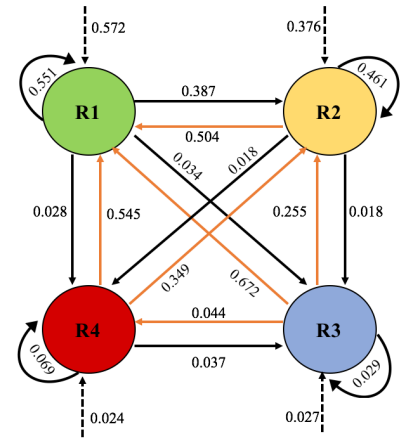

(f) UCLFinal

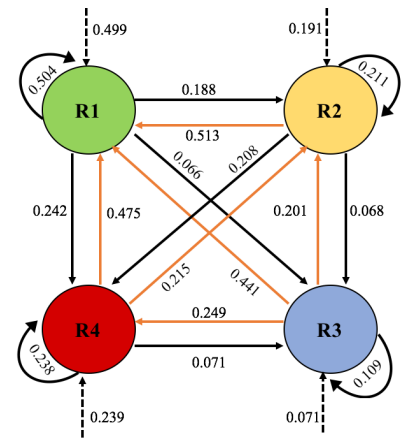

(i) GrenfellTower

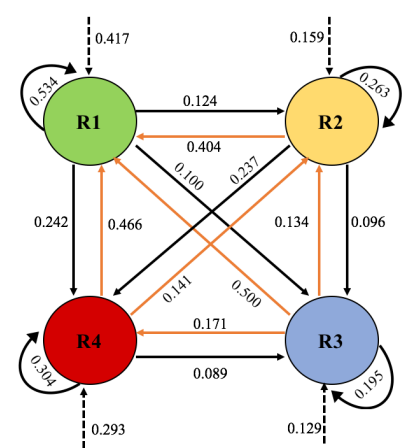

(l) Trumpregrets

Fig. 5. Transition probability of a user over topics in different categories. 
Table 2. Agreement and disagreement by all topics with average transition in Figure 4. $P_{11}$ considers self transition with high positive emotion, i.e., $R 1, P_{i j}$ considers transition from self state to other states, $P(\rightarrow i)$ considers from others state to $R 1$ and $\pi_{i}$ considers initial state.

\begin{tabular}{|l|l|c|c|c|c|}
\hline Dataset & Category & $P_{11}$ & $P_{i j}$ & $P(\rightarrow i)$ & $\pi_{i}$ \\
\hline \#blackmoneydebate & Policy & $\sqrt{ }$ & $\times$ & $\sqrt{ }$ & $\sqrt{ }$ \\
\hline \#brexit & Policy & $\sqrt{ }$ & $\times$ & $\sqrt{ }$ & $\sqrt{ }$ \\
\hline \#AlienCovenant & Movie & $\sqrt{ }$ & $\sqrt{ }$ & $\sqrt{ }$ & $\sqrt{ }$ \\
\hline \#Baahubali2 & Movie & $\times$ & $\times$ & $\sqrt{ }$ & $\times$ \\
\hline \#BadmintonRio2016 & Sport & $\sqrt{ }$ & $\times$ & $\sqrt{ }$ & $\sqrt{ }$ \\
\hline \#UCLFinal & Sport & $\sqrt{ }$ & $\sqrt{ }$ & $\sqrt{ }$ & $\sqrt{ }$ \\
\hline \#SyriaGasAttack & TA & $\times$ & $\times$ & $\sqrt{ }$ & $\times$ \\
\hline \#StockholmAttack & TA & $\times$ & $\times$ & $\sqrt{ }$ & $\times$ \\
\hline \#GrenfellTower & Accident & $\sqrt{ }$ & $\sqrt{ }$ & $\sqrt{ }$ & $\sqrt{ }$ \\
\hline \#UnitedAirlinesAssault & Accident & $\sqrt{ }$ & $\sqrt{ }$ & $\sqrt{ }$ & $\sqrt{ }$ \\
\hline \#MacronPresident & Politics & $\sqrt{ }$ & $\times$ & $\sqrt{ }$ & $\sqrt{ }$ \\
\hline \#Trumpregrets & Politics & $\sqrt{ }$ & $\sqrt{ }$ & $\sqrt{ }$ & $\sqrt{ }$ \\
\hline
\end{tabular}

Figure 4 shows average transition probability from one emotion state to another over the topics in different categories. First, Figure 4 shows the macro average transition probabilities over all topics. It is clearly evident that if a user is in a state with highly positive emotion $R 1$, the probability of staying in the same state in the subsequent tweets from the same user is higher than that of the highly negative emotion state $R 4$ (with probability 0.60 for staying in $R 1$ and 0.31 for staying in $R 4$ ). Further, it can be also seen that if a user makes a transition from one state to another, the user is more likely to move toward the state with highly positive emotion as compared to other states (on average probability $0.43,0.17,0.5$ and 0.15 towards $R 1, R 2, R 3$ and $R 4$, respectively). Interestingly, $R 1$ has got the highest initial transition probability. It means when a random user posts his/her opinion on a random topic, $\mathrm{s}(\mathrm{he})$ is likely to start with highly positive emotion state. However, for the topics like Terror Attack, the observations deviate from the above average pattern.

The above observation may be biased by the nature of the topics/events that we consider in the experimental dataset. Users' emotional states may depend on the nature of the topic under consideration. To understand the topic dependent characteristics, we further investigate topic-wise (Figure 5) transition probability as follows.

- For majority of the topics except in Terror Attack category, like in average case, a user in a highly positive emotional state $(R 1)$ is likely to continue in the same state with higher probability (with probability more than 0.5$)$ as compared to that of the highly negative emotional state $(R 4)$.

- The topics in Terror Attack category show slightly different characteristics where probabilities of a user staying in the highly positive emotion state $(R 1)$ and highly negative emotion state $(R 4)$ are comparable. The probability of staying in $R 4$ is even slightly higher than that of $R 1$.

- Another interesting observation for the topics related to a terror attack is that when users make state transitions, the probabilities of moving towards both $R 1$ and $R 4$ are also comparable.

- For the topics related to Policy, in the majority of the cases users continue to stay in the earlier state (with a probability higher than 0.5 , users continue to take self transition.)

- In the majority of the cases, users in states $R 2$ and $R 3$ are more pron to take the transition to other states than that of $R 1$ and $R 4$. Further, users in $R 3$ are more pron to change state than the users in other states.

- Unlike other topics, for the topics (\#StockholmAttack, \#SyriaGasAttack) Terror Attack category, the initial probability is quite high for region $R 4$. 


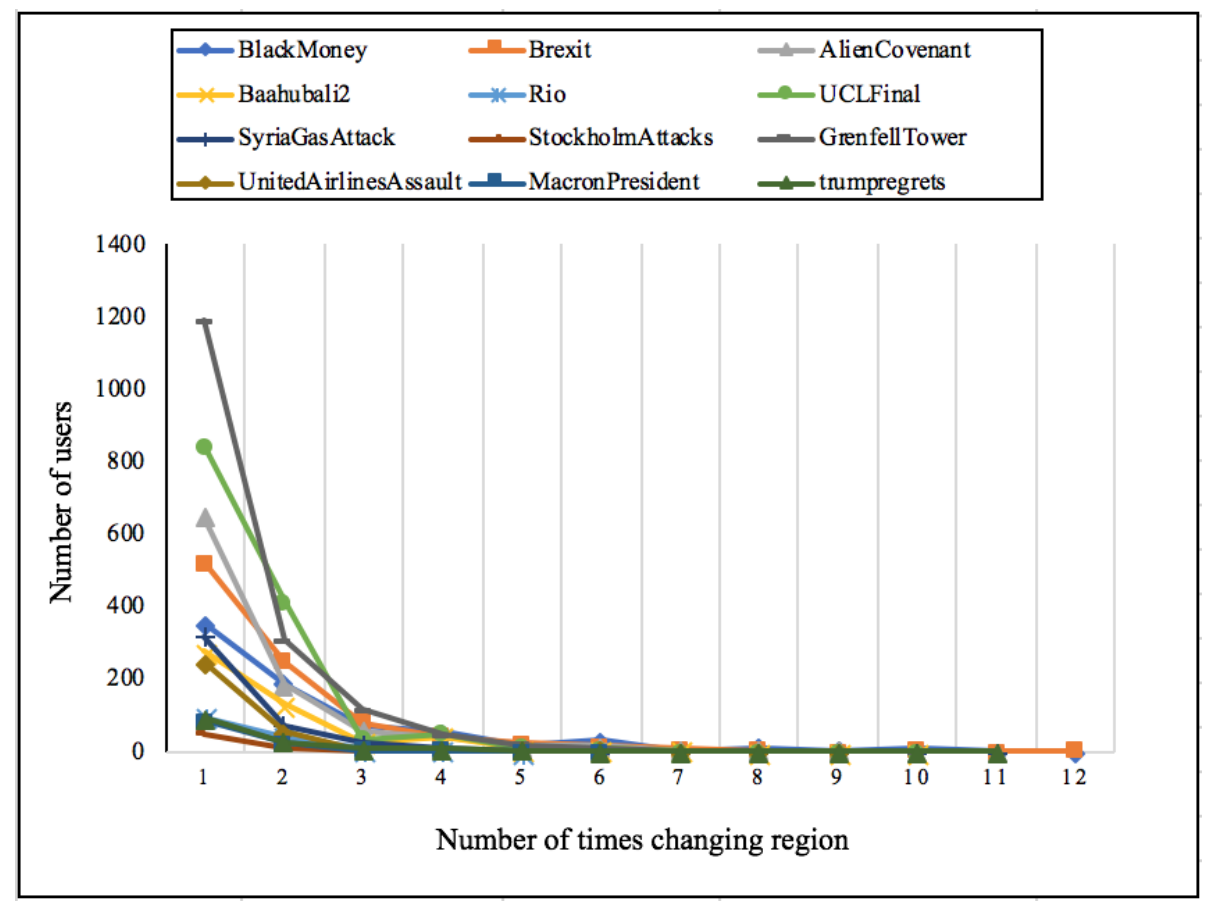

Fig. 6. Frequency of the user changing emotion region across topics

Table 2 summarizes agreement and disagreement of all the topics with that of the observation on average. It clearly shows that except for topics related to terror attacks, the majority of the cases agree with the average observations over all topics. Further, Figure 6 shows the number of times the users have changed their emotion state. We see that, for all the topics, the majority of the users change their emotion state against a topic only once. Comparatively, a very small number of users change their emotion state more than once for the same topic. Further, it is observed that $63 \%$ of users change his/her emotion state at least once against a topic. Users in emotion state $R 3$ has the highest likelihood of changing state with a probability of $32 \%$.

\section{UNDERSTANDING INFLUENCE OF INCOMING TWEETS}

On Twitter user receives messages from other users through various channels like mention, reply, member-list, follower-list, etc. From the studies [Cha et al. 2010], it is noted that the user's opinion is often influenced by the incoming messages (s)he receives. In this section, we attempt to understand the influential characteristics of different channels over the user's opinion by estimating the probability of the incoming emotion state coming through a channel agreeing with the user emotion state present in his post. As for example, given that emotion state $R 1$ of the user and majority of the tweets that the user received, what is the likelihood that the user carrying the majority on an emotion state $R 1$. In this section, we examine three types of social features; (i) incoming tweets with emotion state (i.e., $R 1, R 2, R 3$ and $R 4$ ), (ii) incoming tweets only from community (i.e., InFL, InML and $I n O L$ ), and (iii) incoming tweets only through conversation (InM, InRT, InR and $\operatorname{InH}$ ). From the first features, we attempt to understand, if majority opinion can influence an individual's opinion. The second features try to understand if an individual's opinion can be biased by the opinions coming from the group/community that (s)he belongs to. Lastly, we investigate if responses from the general public on previous posts of an individual 
Table 3. It shows the probability of an individual's emotion state in a post not matching with the emotion state of any of the incoming message

\begin{tabular}{|c|c|c|c|c|c|c|c|c|c|c|c|c|c|}
\hline $\begin{array}{l}\text { Emotion } \\
\text { State }\end{array}$ & 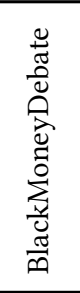 & 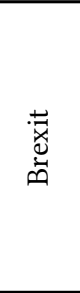 & 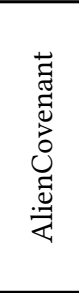 & 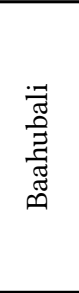 & 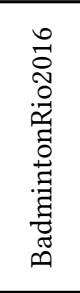 & 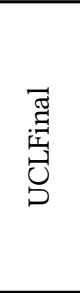 & 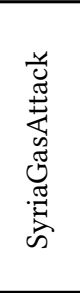 & 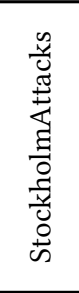 & 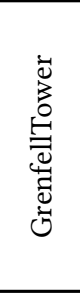 & 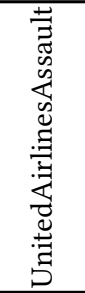 & 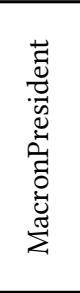 & 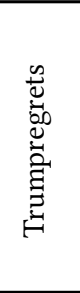 & 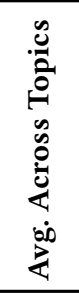 \\
\hline R1 & 0.464 & 0.510 & 0.422 & 0.600 & 0.194 & 0.397 & 0.664 & 0.466 & 0.538 & 0.237 & 0.351 & 0.594 & 0.477 \\
\hline $\mathrm{R} 2$ & 0.830 & 0.758 & 0.668 & 0.565 & 0.813 & 0.639 & 0.824 & 0.883 & 0.790 & 0.707 & 0.845 & 0.725 & 0.760 \\
\hline R3 & 0.903 & 0.888 & 0.898 & 0.819 & 0.974 & 0.976 & 0.970 & 0.755 & 0.917 & 0.918 & 0.925 & 0.858 & 0.896 \\
\hline R4 & 0.793 & 0.833 & 0.890 & 0.949 & 0.937 & 0.977 & 0.483 & 0.570 & 0.740 & 0.804 & 0.651 & 0.760 & 0.798 \\
\hline $\begin{array}{l}\text { Avg. Across } \\
\text { Emotion } \\
\text { State }\end{array}$ & 0.672 & 0.669 & 0.553 & 0.623 & 0.246 & 0.521 & 0.639 & 0.566 & 0.667 & 0.332 & 0.489 & 0.683 & 0.645 \\
\hline
\end{tabular}

influence the individual. We systematically explore the above questions by exploiting the dataset that we prepare in section 3 .

\subsection{Can majority opinions influence individual's opinion?}

To answer this, we examine distribution of the emotion states over all the incoming tweets that an individual receive before he posts his next tweet, and check if the emotion state in his post agrees with any of the emotions in the incoming tweets. To systematically investigate the influential characteristics of the incoming message, we intend to understand the following sub-questions?

5.1.1 When an individual posts an opinion, how likely does his emotion state agree with that of any of the incoming messages? To answer this question, whenever a user post a tweet, we check in how many cases his emotion state matches with the emotion state of the incoming tweets he receives. Table 3 shows the probability of an individual's emotion state when he posts a tweet not matching with the emotion state of the incoming message across different topics. When an individual posts a tweet, if the emotion state of his tweet is matching with the emotion state of any of the incoming tweet then we refer to it as Matching, otherwise UnMatching. The last row of the Table 3 shows the percentage of UnMatching posts for each topic. It clearly shows that except two out of twelve topics, the percentage of UnMatching posts is higher than that of the Matching posts. Across all topics, $64.5 \%$ of the cases, user's posts do not agree with any of the incoming tweet that he receives. It indicates that majority of the user's opinion does not depend on the incoming opinion.

In rows $(R 1, R 2, R 3$, and $R 4)$, it further shows the probability of UnMatching, if user posts a tweet with emotion state $R_{i}, i=1,2,3,4$ i.e., $\operatorname{Pr}\left(U n M a t c h i n g \mid R_{i}\right)=\frac{\operatorname{Pr}\left(R_{i} \mid U n M a t c h i n g\right) \operatorname{Pr}(U n M a t c h i n g)}{\operatorname{Pr}\left(R_{i}\right)}$. It shows that user has higher chances of Matching when he posts a message with positive emotion (i.e., across all topics, the average of $\operatorname{Pr}\left(\right.$ UnMatching $\left.\mid R_{1}\right)$ is smaller than that of $R 2, R 3$, and $\left.R 4\right)$. Similarly, chances of UnMatching is higher when he posts with low positive or high negative emotion state ( $R 3$ or $R 4)$. These observations are true for majority of the topics.

5.1.2 If individual's emotion state matches with incoming emotion, does (s)he agree with majority? To answer this question, we further estimate the probability of user emotion state matching with the majority emotion state of 
the tweets that user receives. Table ??(a) shows the probability of matching with the majority emotion state. In the table, $M_{i}$ denotes $i^{t h}$ majority emotion states i.e., $1^{\text {st }}, 2^{\text {nd }}, 3^{r d}$, and $4^{\text {th }}$ majority. An entry in $R_{i}$ row and $M_{j}$ column in Table ??(a) is the probability that user posts a tweet with emotion $R_{i}$ and the emotion state of $M_{j}$ is also $R_{i}$ i.e., $\operatorname{Pr}\left(M_{j}=R_{i} \mid e(\uparrow m)=R_{i}\right)$ where $e(\uparrow m)$ is the emotion state of the user's outgoing tweet $m$.

The row with total average in Table ?? column (a) shows the probability of agreeing with $M_{i}, i=1,2,3,4$ if user's outgoing post has an agreement with an incoming tweet over all topics across different emotion state. It shows that probability of agreeing with $M_{1}$ is higher than that of $M_{2}, M_{3}$ and $M_{4}$. It means that if the user's emotion state in the tweet that he posted has an agreement with the emotion state of some of the incoming tweets that he received, it is likely to agree with the dominant emotion state among all the tweets he receives.

Further, the $\operatorname{Pr}\left(R_{i}\right)$ column in Table ??(a) shows the probability of a user posting a comment/tweet with the emotion state $R_{i}$. It shows that in majority of the cases user posts tweets with positive emotion $R_{1}$ irrespective of the topics. Overall, $48.3 \%$ percent of the tweets are in $R_{1}$. Interestingly, even for the events like (7) SyriaGasAttack or (8) StockholmAttacks, majority of the tweets are with positive emotion i.e., $43.2 \%$ for (7) SyriaGasAttack and $49.1 \%$ for StockholmAttacks.

For different emotion states, it is observed that when a user posts a tweet with $R 1$, it mostly agrees with dominant emotion in the incoming tweets i.e., $M_{1}$. However, this is not the case when a user posts a tweet with other emotion states (other than $R 1$ ). For example, when a user posts a tweet with $R 3$ emotion state, it agrees mostly with either $M_{3}$ or $M_{4}$. It indicates that a significant number of users do not get influenced by what he receives.

5.1.3 Remarks. Coming back to our earlier question i.e., can majority opinions influence individual's opinion? From the above observations in Table ??(a) i.e., emotion state of a significant number of posts do not agree with the dominant emotion indicates that influential ability of social propaganda on the social network is questionable.

\subsection{Which community channel is more influential?}

In the above section, we have considered all the incoming messages irrespective of the type of channels through which a user receives the messages. However, as indicated in the studies [Cha et al. 2010], different channels may have a different influential pattern. In this section, we investigate the influential characteristics of different channels over the individual opinion. Table ??(b) shows the probability of agreement with the dominant emotion state in each of the community channel namely member-list (ML), following-list (FL) and other-list (OL). An entry in $R_{i}$ row and $M L$ column in Table ??(b) is the probability that user posts a tweet with emotion $R_{i}$ and majority of the tweets coming from $M L$ have emotion state $R_{i}$, i.e., percentage of agreement with the dominant state in $M L$. Similarly, entries at $F L$ and $O L$ represent the percentage of agreement with their respective dominant state.

It is evident from Table ??(b), $M L$ has the highest probability of agreement as compared to $F L$ and $O L$ for all the emotion state $R_{i}, i=1,2,3,4$. It means that message coming from $M L$ has a higher potential for influencing users than the tweets coming from $F L$ and $O L$. From the rows with total average in Table ??(b), it is observed that $M L$ has $76 \%$ of agreements, whereas $F L$ has only $22 \%$. An interesting observation is that, though $M L$ dominates $F L$ for almost all the topics, the topics like BlackMoneyDebate, Brexit and BadmintonRio2016 have comparable distribution between $M L$ and $F L$. This is due to the fact that popular tweets propagate multiple times from the source by retweeting throughout the network. A study [Watts and Dodds 2007] indicates that retweeting is a powerful mechanism in the social network where a group of users re-post the same tweet.

\subsection{Which conversational channel is more influential?}

In the previous section, it is observed that tweets coming from $M L$ has higher potential of influencing user's opinion. Further, tweets might be coming through different conversational channels like hashtags $(H)$, retweet $(R T)$, mention $(M)$ and reply $(R)$. In this section, we further investigate influential characteristics of these conversational 


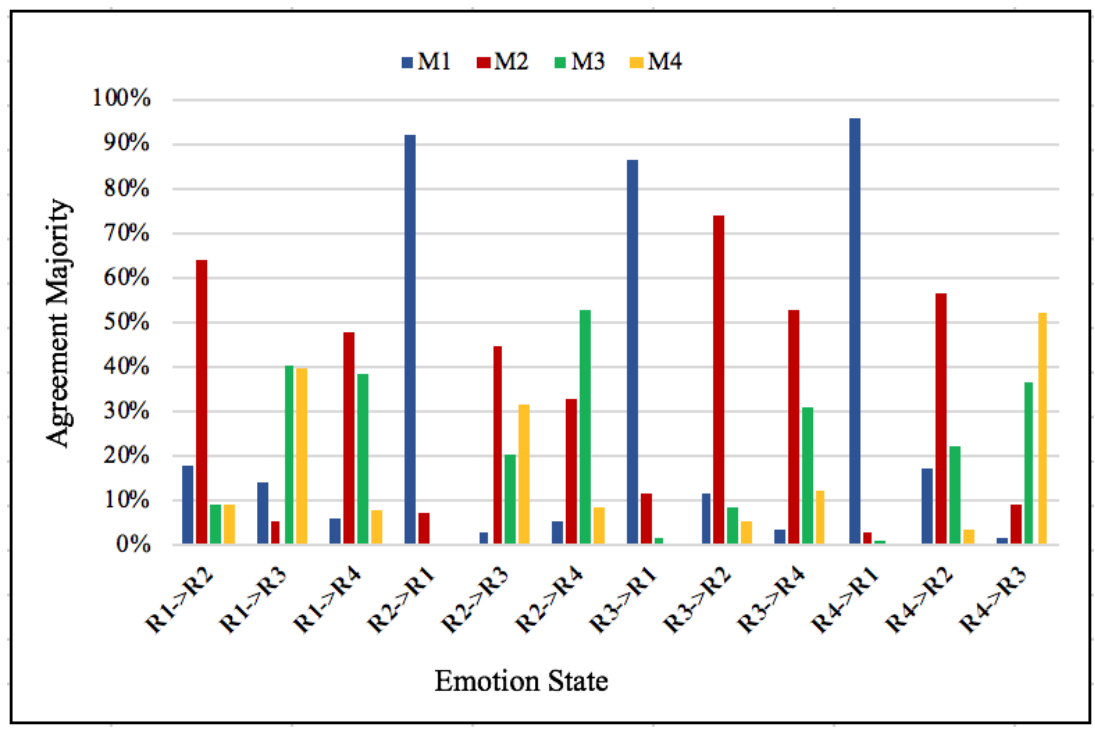

Fig. 7. Average probabilities of emotional agreement over all topics

channels. Table ??(c) shows the probability of agreement with the dominant emotion state in each of the above conversational channel. An entry in $R_{i}$ row and conversational channel in Table ??(c) is the probability that user posts a tweet with emotion $R_{i}$ and majority of the tweets coming from the channel also have emotion state $R_{i}$.

In almost all the cases over all the topics, $R T$ dominants $H, R$ and $M$. It means that tweets coming from $R T$ have higher potential for influencing user's opinion than the tweets coming from others $(H, R, M)$. Similar observations have also been reported in the study [Suh et al. 2010b]. Among $H, R$ and $M, H$ has higher potential. From the row with total average in Table ??(c), it is observed that $R T$ contributes in $81 \%$ of the agreements, $H$ contributes in $12 \%$, and rest from $M$ and $R$.

\section{CHARACTERISTICS OF INCOMING TWEETS TOWARDS STATE TRANSITION}

In section 4 , we observe that $63 \%$ of users change their opinions towards a topic at least once. This section investigates the distribution of the incoming tweets that an individual receives at the time of changing his/her emotion state while posting a tweet against a topic. It will help us to understand possible causal influence from different channels through which an individual receives incoming tweets. Like in section 5 , this section also considers the same three types of cases; whole incoming tweets, community channels and conversational channels.

\subsection{State Transition Vs Incoming dominant emotion}

Why does an individual change his opinion from his/her earlier emotion state against a topic? Has it been influenced by the emotion state of the majority opinion? To answer these questions, we study the distribution of the emotion state of all the incoming tweets that an individual receives before posting his/her next tweet with a different emotion state (emotion state different from his/her previous tweet). Figure 7 shows the distribution of the emotion state of the incoming tweets across different dominant emotion states for each possible state transition.

In Figure 7, the emotion transitions of a user is represented as $R_{i} \rightarrow R_{j}$ (where $i, j=1,2,3,4$ ). For each $R_{i} \rightarrow R_{j}$ transition, we have shown its agreement with the distribution of the incoming emotion state. A bar chart with $M_{k}$ (where $k=1^{s t}, 2^{n d}, 3^{r d}$, and $4^{t h}$ ) against a state transition $R_{i} \rightarrow R_{j}$ represents the number of the cases (in 


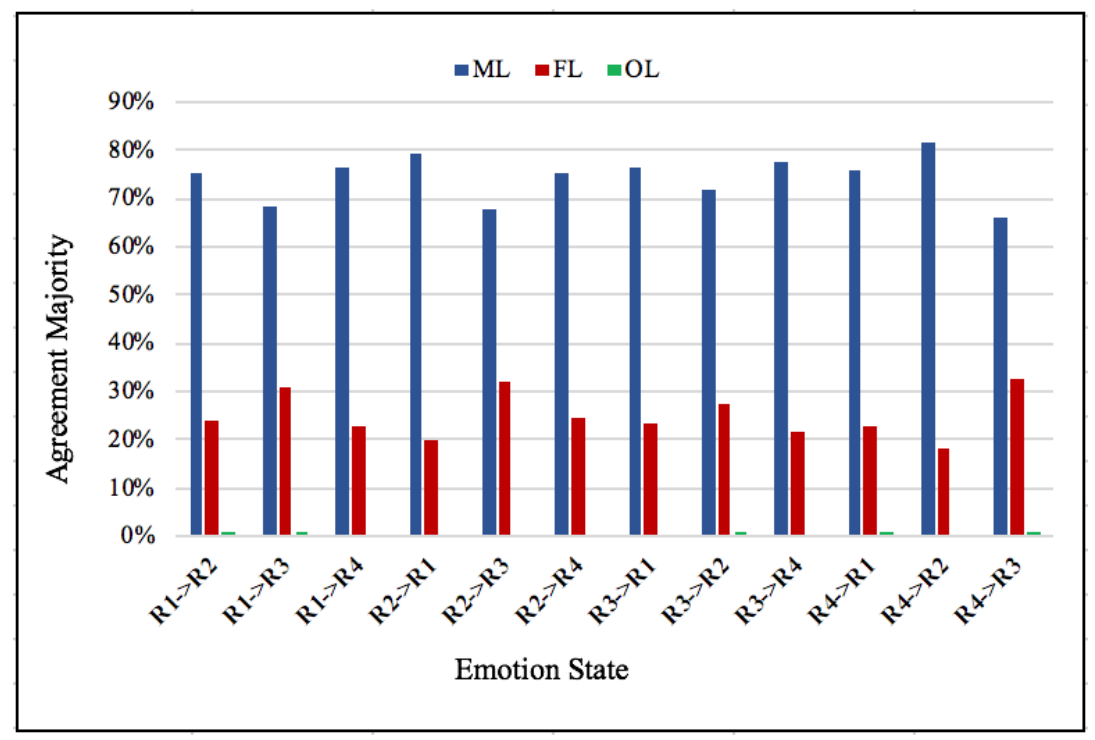

Fig. 8. Average probabilities of topological agreement over all topics

percentage) in which the emotion state changes from $R_{i}$ to $R_{j}$, and emotion state of the $k^{\text {th }}$ majority incoming tweet is $R_{j}$.

A user's agreement with $1^{\text {st }}$ majority i.e., $M_{1}$ at the time of state transition (change in emotion state of the user i.e., $R_{i} \rightarrow R_{j}, i \neq j$ ) potentially reflects possible influenced of changing state from the dominant incoming emotion. Similarly, a disagreement with the dominant emotion state at the time of changing the state of a user may also indicate a user's ability to make his/her own opinion (not biased by the dominant incoming emotion). Figure 7 shows two interesting observations. Whenever users change his emotion state to extreme positive from any other state, it always has an agreement with the majority $\left(M_{1}\right)$ i.e., $R_{i} \rightarrow R_{1}, i \neq 1$. However, for any other changes where $R_{i} \rightarrow R_{j}, j \neq 1$, users agreement with $M_{1}$ is very low. It potentially means that sharing positive emotion is more general, and sharing negative emotion is more personal. It is interesting to see that transition to extreme negative has agreement mostly with $2^{\text {nd }}$ and $3^{r d}$ dominant emotions, not with the majority. Figure 7 shows that when users change their emotion state from $R 4 \rightarrow R 1$ and $R 1 \rightarrow R 4$, the percentage of agreements with dominant incoming emotion are $96 \%$ and $6 \%$ respectively. We can also see that the percentage of agreement with dominant incoming emotion while the transition from low positive $R 3$ to high positive $R 1$ is higher than that of $R 1$ to $R 3$ (i.e. $87 \%$ and $14 \%$ ).

From the above observations, it is evident that when a user changes his/her emotion state to non-positive states (i.e., $R j, j \neq 1$ ), the user tends to share personal opinion. How large is this proportion? In our dataset, $40 \%$ of the state transitions do not agree with $M_{1}$ and $41 \%$ of these transitions belong to a non-positive state transition. Further, how likely a user who had an agreement with the majority tends to change his/her state? It is observed from the dataset that in $2 \%$ of the cases a user tends to change his/her state from agreement to disagreement with the $M_{1}$ i.e., $R_{i} \rightarrow R_{j}, R_{i}=M_{1}, R_{j} \neq M_{1}$. It shows a significant proportion of the users are not influenced by incoming emotions. 


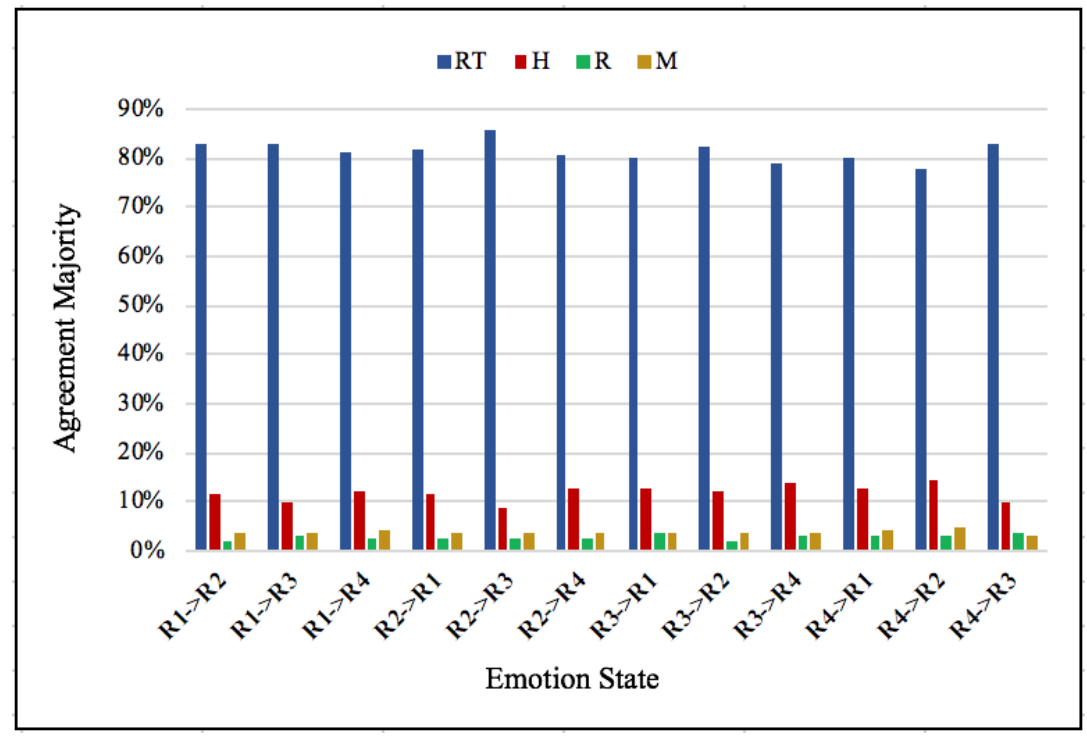

Fig. 9. Average probabilities of conversational agreement over all topics

\subsection{Which community channel is more influential during emotion state transition?}

In the previous section, we have investigated over entire incoming tweets irrespective of the channels through which they receive the incoming tweets. In this section, we study the emotion distribution of the incoming tweets with respect to community channels i.e., member list, following, and others. Like in section 6.1, we estimate the probability of user emotion state matching with a different channel through (s)he receives during emotion transition. We calculate the total average probability of agreeing with the different channels over all topics in different categories. In Figure 8, the emotion transition refers to $R_{i} \rightarrow R_{j}$, where $i, j=1,2,3,4$ and for each $R_{i} \rightarrow R_{j}$ transitions, we have shown its agreement with majority for each community channel i.e., $M L$ for member list, $F L$ for following and $O L$ for others. Each bar corresponding to a channel for a given transition $R_{i} \rightarrow R_{j}$ represents the percentage of its emotional agreement with the dominant emotional state in each channel i.e., the dominant emotion state is $R_{j}$.

Figure 8 shows that if an individual changes their emotion state from one region to another, the average majority agreement of the member-list channel is always higher than other channels (i.e. 74\%). It is true for all the $R_{1} \rightarrow R_{j}, i \neq j$ pairs. From this observation, it is evident that $M L$ has maximum contribution in causing the state change. Interestingly emotion agreement with tweets coming from other $(O L)$ channels is negligible. It shows that users are mostly influenced by tweets coming from either member list and following. Between member-list and following-list, member-list significantly dominants the following-list.

\subsection{Which conversational characteristics is more influential during emotion state transition?}

Individuals also receive tweets through conversational characteristics like Re-tweet, Hashtag, Reply and Mention. Which one of these is more influential in causing state transition? To acknowledge the above question, we investigate of distribution of dominant emotion states over these channels in Figure 9. We calculate the total average probability of agreeing with different conversational characteristics over all topics in different categories. Like above, each bar in Figure 9 shows the percentage of the instances of agreement with the majority in each 
characteristic against different possible state transition $R_{i} \rightarrow R_{j}, i \neq j$. It is evident from the figure that if an individual changes their emotion state from one region to another, then the retweet contribute the most with about $81 \%$ on average over all transitions. Further, Hashtags contribute about $10 \%$. The contribution from the reply and mention are negligible.

\section{CONCLUSION}

This paper analyzes the emotion dynamics of users while posting public opinion through Twitter. This study has considered a dataset consisting of 17.65 million tweets with about 69.36k users over 12 different topics. From this dataset, emotion dynamics of $12.91 \mathrm{k}$ users who have posted at least two tweets have been studied. Analysis has been investigated from three different perspectives; the user's emotion transition, the influence of the emotion of public opinion on an individual's opinion, social parameters causing a change in an individual's opinion. First, we observe that $63 \%$ of the user change their opinion against a topic. People who share positive emotion against topics are likely to stay in the same emotional state in his/her subsequent tweets. Users in the highly negative state have the highest probability of changing state. If users change their emotional state, the probability of changing towards the highly positive state is higher than the probability of changing towards the highly negative state. It is observed that tweets coming from the member community have higher influential capability than the tweets coming from the follower community and other sources. Further, it is also observed that retweeted tweets can influence users higher than hashtag, reply and mention.

In order to investigate systematically, we perform three different experiments over several categorical social topics/events on Twitter. First, we perform emotion state transition over entire conversation. By focusing on user emotion transition helps others to find someone's indisputable interest on the topic in future. From the transition, we infer that people shown positive opinion into their conversation except terrorism attack topics. We also infer that at the initial stage people shows high positive emotion and while continuing their conversation, most people going towards high positive direction. Second, we perform an analysis to understand influential characteristics of different channels over user's opinion by estimating of the incoming emotion state coming through a channel. From this analysis, we infer emotion state of a significant number of post do not influence with the dominant emotion on social network. Finally, we investigate the influential characteristics towards state transition by approaching three types of cases (whole incoming tweets, community channels and conversational characteristics). From this investigation, we infer that majority people agree with positive emotion when users change their emotion state from negative to positive. As a result, it is significant that majority people of state transition potentially reflects possible influenced of changing state from the dominant incoming emotion.

\section{ACKNOWLEDGMENTS}

The work described in this paper was carried out in the OSiNT Lab (https://www.iitg.ac.in/cseweb/osint/), Indian Institute of Technology Guwahati, India. The creation of the Dataset used in this study was partly supported by the Ministry of Information and Electronic Technology, Government of India.

\section{REFERENCES}

Saifuddin Ahmed, Kokil Jaidka, and Jaeho Cho. 2017. Tweeting India's Nirbhaya protest: a study of emotional dynamics in an online social movement. Social Movement Studies 16, 4 (2017), 447-465.

Anthoniraj Amalanathan and S Margret Anouncia. 2017. Influence of human emotion expressed through social networks in viral marketing. International fournal of Business Information Systems 26, 1 (2017), 15-32.

Christophe Andrieu, Nando De Freitas, Arnaud Doucet, and Michael I Jordan. 2003. An introduction to MCMC for machine learning. Machine learning 50, 1-2 (2003), 5-43.

Theo Araujo, Peter Neijens, and Rens Vliegenthart. 2017. Getting the word out on Twitter: The role of influentials, information brokers and strong ties in building word-of-mouth for brands. International fournal of Advertising 36, 3 (2017), 496-513.

Jonah Berger. 2011. Arousal increases social transmission of information. Psychological science 22, 7 (2011), $891-893$. 
Bin Bi, Yuanyuan Tian, Yannis Sismanis, Andrey Balmin, and Junghoo Cho. 2014. Scalable topic-specific influence analysis on microblogs. In Proceedings of the 7th ACM international conference on Web search and data mining. ACM, 513-522.

Johan Bollen, Huina Mao, and Xiaojun Zeng. 2011. Twitter mood predicts the stock market. fournal of computational science 2, 1 (2011), 1-8. MM Bradley and PJ Lang. 2010. Affective norms for English Words (ANEW): Affective ratings of words and instruction manual. University of Florida, Gainesville. FLTechnical Report C-2 (2010).

Meeyoung Cha, Hamed Haddadi, Fabricio Benevenuto, and P Krishna Gummadi. 2010. Measuring user influence in twitter: The million follower fallacy. ICWSM 10, 10-17 (2010), 30.

Wei Chen, Chi Wang, and Yajun Wang. 2010. Scalable influence maximization for prevalent viral marketing in large-scale social networks. In Proceedings of the 16th ACM SIGKDD international conference on Knowledge discovery and data mining. ACM, 1029-1038.

Niko Colneriĉ and Janez Demsar. 2018. Emotion Recognition on Twitter: Comparative Study and Training a Unison Model. IEEE Transactions on Affective Computing (2018).

Jean MR Costa, Rahmtin Rotabi, Elizabeth L Murnane, and Tanzeem Choudhury. 2015. It is not only about Grievances-Emotional Dynamics in Social Media during the Brazilian Protests. In Proceedings of the Ninth International AAAI Conference on Web and Social Media. 594-597.

Zhaoyun Ding, Yan Jia, Bin Zhou, Jianfeng Zhang, Yi Han, and Chunfeng Yu. 2013. An influence strength measurement via time-aware probabilistic generative model for microblogs. In Asia-Pacific Web Conference. Springer, 372-383.

Zhaoyun Ding, Hui Wang, Liang Guo, Fengcai Qiao, Jianping Cao, and Dayong Shen. 2015. Finding Influential Users and Popular Contents on Twitter. In International Conference on Web Information Systems Engineering. Springer, 267-275.

Lisa Feldman Barrett and James A Russell. 1998. Independence and bipolarity in the structure of current affect. fournal of personality and social psychology 74, 4 (1998), 967.

Emilio Ferrara and Zeyao Yang. 2015. Measuring emotional contagion in social media. PloS one 10, 11 (2015), e0142390.

Qi Gu, Jr Eugene Santos, and Eunice E Santos. 2013. Modeling opinion dynamics in a social network. In Proceedings of the 2013 IEEE/WIC/ACM International foint Conferences on Web Intelligence (WI) and Intelligent Agent Technologies (IAT)-Volume 02. IEEE Computer Society, 9-16.

Maryam Hasan, Emmanuel Agu, and Elke Rundensteiner. 2014. Using hashtags as labels for supervised learning of emotions in twitter messages. In ACM SIGKDD Workshop on Health Informatics, New York, USA.

Robert Hillmann and Matthias Trier. 2012. Dissemination patterns and associated network effects of sentiments in social networks. In IEEE International Conference on Advances in Social Networks Analysis and Mining, ASONAM, 2012. 511-516.

Maria Karakiza. 2015. The Impact of Social Media in the Public Sector. Procedia - Social and Behavioral Sciences 175 (02 2015). https: //doi.org/10.1016/j.sbspro.2015.01.1214

Suin Kim, JinYeong Bak, and Alice Haeyun Oh. 2012. Do You Feel What I Feel? Social Aspects of Emotions in Twitter Conversations.. In ICWSM.

Haewoon Kwak, Changhyun Lee, Hosung Park, and Sue Moon. 2010. What is Twitter, a social network or a news media?. In Proceedings of the 19th international conference on World wide web. ACM, 591-600.

Yung-Ming Li and Ya-Lin Shiu. 2012. A diffusion mechanism for social advertising over microblogs. Decision Support Systems 54, 1 (2012), 9-22.

Panagiotis Liakos, Katia Papakonstantinopoulou, Michael Sioutis, Konstantinos Tsakalozos, and Alex Delis. 2016. Pinpointing Influence in Pinterest.. In SocInf@IfCAI. 26-37.

Seth A Myers, Chenguang Zhu, and Jure Leskovec. 2012. Information diffusion and external influence in networks. In Proceedings of the 18th ACM SIGKDD international conference on Knowledge discovery and data mining. ACM, 33-41.

Debashis Naskar, Sidahmed Mokaddem, Miguel Rebollo, and Eva Onaindia. 2016. Sentiment Analysis in Social Networks through Topic modeling.. In LREC.

Hung T Nguyen, Preetam Ghosh, Michael L Mayo, and Thang N Dinh. 2017. Social influence spectrum at scale: Near-optimal solutions for multiple budgets at once. ACM Transactions on Information Systems (TOIS) 36, 2 (2017), 14.

Finn Årup Nielsen. 2011. A new ANEW: Evaluation of a word list for sentiment analysis in microblogs. In ESWC Workshop on 'Making Sense of Microposts': Big things come in small packages, MSM, 2011. 93-98.

Brendan O'Connor, Ramnath Balasubramanyan, Bryan R Routledge, and Noah A Smith. 2010. From tweets to polls: Linking text sentiment to public opinion time series. In Fourth International AAAI Conference on Weblogs and Social Media.

Aditya Pal and Scott Counts. 2011. Identifying topical authorities in microblogs. In Proceedings of the fourth ACM international conference on Web search and data mining. ACM, 45-54.

Sancheng Peng, Guojun Wang, and Dongqing Xie. 2017. Social influence analysis in social networking big data: opportunities and challenges. IEEE Network 31, 1 (2017), 11-17.

James A Russell. 1980. A circumplex model of affect. fournal of personality and social psychology 39, 6 (1980), 1161-1178.

Diego Saez-Trumper, Giovanni Comarela, Virgílio Almeida, Ricardo Baeza-Yates, and Fabrício Benevenuto. 2012. Finding trendsetters in information networks. In Proceedings of the 18th ACM SIGKDD international conference on Knowledge discovery and data mining. ACM, 1014-1022. 
Juan Shi, Ping Hu, Kin Keung Lai, and Gang Chen. 2018. Determinants of users? information dissemination behavior on social networking sites: an elaboration likelihood model perspective. Internet Research 28, 2 (2018), 393-418.

Arlei Silva, Sara Guimarães, Wagner Meira Jr, and Mohammed Zaki. 2013. ProfileRank: finding relevant content and influential users based on information diffusion. In Proceedings of the 7th Workshop on Social Network Mining and Analysis. ACM, 2.

Stefan Stieglitz and Linh Dang-Xuan. 2013. Emotions and information diffusion in social media?sentiment of microblogs and sharing behavior. fournal of management information systems 29, 4 (2013), 217-248.

Bongwon Suh, Lichan Hong, Peter Pirolli, and Ed H Chi. 2010a. Want to be retweeted? large scale analytics on factors impacting retweet in twitter network. In 2010 IEEE Second International Conference on Social Computing. IEEE, 177-184.

Bongwon Suh, Lichan Hong, Peter Pirolli, and Ed H. Chi. 2010b. Want to be Retweeted? Large Scale Analytics on Factors Impacting Retweet in Twitter Network. In (IEEE) International Conference on Privacy, Security, Risk and Trust, PASSAT 2010, Minneapolis, Minnesota, USA, 2010. 177-184. https://doi.org/10.1109/SocialCom.2010.33

Beiming Sun and Vincent TY Ng. 2013. Identifying influential users by their postings in social networks. In Ubiquitous social media analysis. Springer, 128-151.

Jie Tang and Acm Fong. 2013. Sentiment diffusion in large scale social networks. In IEEE International Conference on Consumer Electronics, ICCE, 2013. 244-245

Ali Vardasbi, Heshaam Faili, and Masoud Asadpour. 2017. SWIM: Stepped weighted shell decomposition influence maximization for large-scale networks. ACM Transactions on Information Systems (TOIS) 36, 1 (2017), 6.

Yanhao Wang, Yuchen Li, Ju Fan, and Kian-Lee Tan. 2018. Location-aware influence maximization over dynamic social streams. ACM Transactions on Information Systems (TOIS) 36, 4 (2018), 43.

Duncan J Watts and Peter Sheridan Dodds. 2007. Influentials, networks, and public opinion formation. Fournal of consumer research 34, 4 (2007), 441-458.

Brian E Weeks, Alberto Ardèvol-Abreu, and Homero Gil de Zúñiga. 2017. Online influence? Social media use, opinion leadership, and political persuasion. International fournal of Public Opinion Research 29, 2 (2017), 214-239.

Jianshu Weng, Ee-Peng Lim, Jing Jiang, and Qi He. 2010. Twitterrank: finding topic-sensitive influential twitterers. In Proceedings of the third ACM international conference on Web search and data mining. ACM, 261-270.

Gadi Wolfsfeld, Elad Segev, and Tamir Sheafer. 2013. Social media and the Arab Spring: Politics comes first. The International fournal of Press/Politics 18, 2 (2013), 115-137.

Shaozhi Ye and S Felix Wu. 2010. Measuring message propagation and social influence on Twitter. com. In International Conference on Social Informatics. Springer, 216-231.

Michelle SM Yik, James A Russell, and Lisa Feldman Barrett. 1999. Structure of self-reported current affect: Integration and beyond. Fournal of personality and social psychology 77, 3 (1999), 600.

Jinxue Zhang, Rui Zhang, Jingchao Sun, Yanchao Zhang, and Chi Zhang. 2016. Truetop: A sybil-resilient system for user influence measurement on twitter. IEEE/ACM Transactions on Networking 24, 5 (2016), 2834-2846.

Yuchi Zhang, Wendy W Moe, and David A Schweidel. 2017. Modeling the role of message content and influencers in social media rebroadcasting. International fournal of Research in Marketing 34, 1 (2017), 100-119.

Cai-Nicolas Ziegler and Georg Lausen. 2005. Propagation models for trust and distrust in social networks. Information Systems Frontiers 7 , 4-5 (2005), 337-358. 